the number of females impregnated.

Elephant seals (Mirounga angustirostris) breed communally on selected small islands. Usually these are greatly overcrowded while other islands remain unburdened. In a six year study on Año Nuevo Island, California, Le Boeuf has focused attention on the small number of dominant males, usually fewer than one third of all resident males, which are responsible for the majority of copulations ( $\mathrm{Am}$. Zool. 14, 163; 1974). Copulation frequency is directly related to success in competition between males for hierarchical accolade with alpha bulls being responsible for up to $49 \%$ of all matings. The Año Nuevo population is increasing, presumably due to subtle habitat changes, and with this increase is a decrease in the alphas' share of copulations. But there does seem to be some density dependent regulation in force. Le Boeuf in 1972 showed that a major source of pup mortality, up to $43 \%$ of all pups born, was the fighting of adult males (Behaviour, 41, 1). Death to the pups was the result of being trampled upon by the bulls which weigh up to three tons. Crude though this mechanism may be, it is certainly effective. Social unrest among the males reduces the number of young produced by increasing infant mortality.

Some of the consequences of lek life in the Uganda kob antelope (Adenota $k o b)$ were reported in these columns a few weeks ago $(\mathbf{2 5 2}, \mathbf{3 4 5}$; 1974). More than 15 years of study of the population in the Torro game reserve has revealed that there is a strong attachment to the home lek and adults of both sexes traditionally return home to breed (Buechner and Roth Am Zool., 14, 145; 1974). Only dominant males hold mating territories within the leks and, since clandestine matings are rare, they alone mate with females. There seems to be some limit to the number of territories in each lek, but what controls this is not known. Since rinderpest exterminated European cattle in this area kob populations have been increasing and a few additional leks are being formed. Population increase does not appear to be achieved by the establishment of additional territories within leks, but by the creation of additional leks.

Amid a welter of fascinating detail, these three studies reveal a couple of fundamental principles. First, they point out that Darwin's observations, sensu stricto, are incorrect because males fight not for females directly but for a conventional reward which itself confers the right to mate. Second, they show that social dominance is the key to conventional reward and hence to frequency of copulation. Additionally Robel's studies show that social stability between males is a major factor governing the number of females fecundated and in Le Boeuf's study social interaction strongly influences infant survival.

Neither Lack's nor Wynne-Edwards's disquisitions can be proved or disproved by these studies although there is strong prima facie evidence that the behaviour of males has a regulatory function on the number of new individuals brought into the population. What has not been shown is whether leks serve primarily to census populations and their resources or to provide an attractive meeting, and mating, place. More long term studies may help to reveal the complications thrown in by such factors as overall population increase and so lead towards a correct interpretation of the function of leks. They may also, as a sideline help to indicate the area in which the regulation of human populations went awry.

\section{Adapting to a chilly future}

\author{
from Peter D. Moore
}

IF "The Threat of Ice" (see Nature, 252,$216 ; 1974$ ) is a real one, it may be salutary to learn how a variety of organisms adapt to cold. The adaptations of plants, which cannot migrate, must be physiological; they are also seasonal, in all but the most severe arctic and alpine climates. It has long been realised that the ability of many plants to tolerate cold varies with their recent temperature experiences (Levitt, Handb. Pflanzen physiol., II, 632; 1956); this knowledge has been used in the horticultural practice of frost hardening. Riedmüller-Schölm (Flora, Jena, 163,$230 ; 1974$ ) has recently examined the low temperature resistance of many Alaskan plants at monthly intervals

\title{
Another function for juvenile hormone
}

ONE attraction of the hormonal system of insects as a medium for the study of endocrinology has lain in the apparent simplicity of the range of hormones involved. As knowledge grows complexity increases. The diversity and subtlety of the effects of the juvenile hormone seem to militate against the formulation of any unitary theory of the control of metamorphosis. But a new factor has come to light in the course of studies by Nijhout and Williams (J. exp. Biol., 61, 481 and 493 ) on the tobacco hornworm Manduca. Throughout the early larval stages of Manduca there seems to be a continuing cycle of secretion of the brain hormone (the prothoracic-otropic hormone: PTTH) which induces the discharge of ecdysone by the prothoracic gland and of juvenile hormone from the corpus allatum, which ensures the maintenance of larval characters. But in the fifth or final larval stage the scene changes: feeding during this stage is more prolonged and when moulting does take place the larva suffers metamorphosis to the pupa.

One thing can be predicted from existing knowledge: the brain must not release PTTH while a large amount of juvenile hormone is circulating, or another larval stage will be produced. What is peculiar about the final larval stage, according to the results of Nijhout and Williams, is that the high titre of juvenile hormone inhibits release of PTTH by the brain. But when the larva reaches a certain weight (about $5 \mathrm{~g}$ ) the corpora allata ceases to secrete the juvenile hormone. Within about $24 \mathrm{~h}$ the juvenile hormone is cleared from the haemolymph and this results in the brain being rendered competent to release PTTH during the ensuing photoperiod. Growth continues for several days to give a final weight of about $8 \mathrm{~g}$. The gut contents are then discharged and the behavioural and developmental changes that lead to pupation are set in train.

These observations provide an informative description of the hormonal changes during the last larval stage in Lepidoptera; and they illustrate a new function for the juvenile hormone. The failure of the normal arrest in juvenile hormone secretion is apparently responsible for the developmental arrest or diapause in the mature larvae of some Lepidoptera. Fukaya and Mitsuhashi and more recently Chippendale and Yin have stressed the importance of juvenile hormone in inducing larval dormancy. The onset and persistence of diapause in mature larvae can now be accounted for by a functional failure of the normal mechanism which inactivates the corpora allata of non-diapausing larvae.

The turning off of the corpus allatum secretion not only permits the morphological change of metamorphosis; it now seems to set in motion in caterpillars the endocrine events that lead to the moult itself. But what turns off the corpus allatum? Some environmental stimulus? Some proprioceptive stimulus? Or the passage of time? 